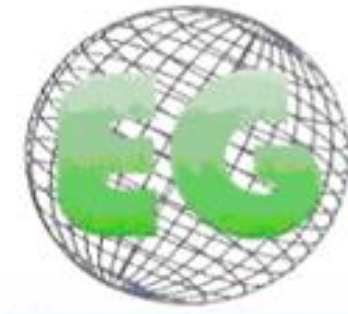

$N^{\circ} 40$

\title{
El uso de Twitter en los XVII y XVIII Encuentros Internacionales de Investigación en Cuidados
}

\author{
Use of Twitter at 17th and 18th International Nursing Research Conferences
}

\section{*Ovalle-Perandones, María-Antonia **Navas-Martín, Miguel-Ángel}

\author{
*Universidad Carlos III de Madrid. Facultad de Humanidades, Comunicación y Documentación. \\ Departamento de Biblioteconomía y Documentación. E-mail: antonia.ovalle@uc3m.es **|nstituto de \\ Salud Carlos III. Unidad de Investigación en Cuidados de Salud (Investén). Madrid. España.
}

Palabras clave: Twitter; Congresos; Redes sociales; Investigación en Cuidados

Keywords: Twitter; Conferences; Social Networks; Nursing Research

\section{RESUMEN}

El objeto de este artículo es proponer una hoja de ruta para analizar el uso de la red social Twitter durante los días en los que se celebra un congreso. La principal motivación se fundamenta en la poca práctica de realizar esta tarea en el contexto español, sí es habitual hacerlo en congresos internacionales, con especial énfasis en el campo de los encuentros en ciencias de la salud. Con la tecnología propia de Opileak se monitorizaron todos los mensajes difundidos en la red social Twitter, durante los cuatro días del Encuentro Internacional de Investigación en Cuidados y que son organizados por la unidad Investén del Instituto de Salud Carlos III. El proceso se realizó tras haber finalizado las conferencias celebradas en los años 2013 y 2014, contando cada una con su propio hashtag: \#inves13 e \#inves14. Los resultados demuestran que los usuarios tienden a retuitear más con el paso del tiempo que a emitir tuits originales; los días centrales del congreso también lo son en cuanto a la actividad en la red social; los usuarios toman diferentes roles siendo algunos buenos informadores y otros estando bien informados; las palabras que utilizan son un reconocimiento a las ciudades donde se celebran los encuentros, a la temática del encuentro y a su carácter internacional. La organización de cualquier encuentro debe realizar este tipo de análisis para descubrir el impacto del evento en un canal de comunicación 2.0

\section{ABSTRACT}

The aim of this paper is to propose a roadmap to analyze the use of the Twitter social network during the days when a conference is held. The main reason is the lack of practice to perform this task in the Spanish context, against the habit of doing at international conferences, with special emphasis in Health Sciences. With the Opileak technology, all messages were monitored on the social network Twitter during four days of the International Nursing Research Conferences, organized by Carlos III Healthcare Institute's, Nursing and Healthcare Research Unit (Investén-ISCIII). The process was made when the conferences were closed in the years 2013 and 2014, both had their own hashtags: \#inves13 and \# inves14. The results show that users tend to retweet more over time than original tweets; the main days of the conference have also the highest activity on the social network; users take different roles, being 
some good informers or well-informed users; the words used are a tribute to the cities where the conferences are held, the theme of the conferences and their international character. This type of analysis should perform by the organization of any meeting to discover the event impact on a communication channel 2.0

\section{INTRODUCCIÓN}

Desde que en 2006 se creó el servicio de microblogging Twitter, más popular tras la conferencia South by SouthWerst (SWSX) en 2007, los procesos de comunicación de la vida habitual han visto modificada su dinámica ${ }^{(1)}$. Su principal característica es el límite de caracteres, hasta 140, con los que los usuarios pueden describir brevemente su estado y automáticamente éste se difunde a aquellos usuarios a los que están conectados, accediendo a la red desde cualquier dispositivo conectado a Internet.

Esos procesos, que forman parte de la conocida como comunicación $2.0^{(2)}$, también han tenido su impacto en los numerosos encuentros científicos que periódicamente se celebran entre profesionales de un sector profesional. En todas las especialidades son importantes, si bien las relaciones entre los profesionales sanitarios tienen múltiples beneficios para el buen funcionamiento de las organizaciones ${ }^{(3)}$ y como efecto secundario, de las personas que forman parte de ellas y de los pacientes.

En la fase en la que no existían redes sociales, a la que se puede denominar como de pre-redes sociales, cuando se organizaba un congreso se le daba la mayor difusión posible entre los potenciales asistentes: profesionales, estudiantes, docentes, investigadores, por mencionar algunos. En ese momento la difusión se realizaba fundamentalmente con un díptico, tríptico o cartel en soporte papel. A finales del siglo pasado comenzaron a irrumpir otras posibilidades como Internet o el correo electrónico, y con ellas los organizadores tuvieron la capacidad de identificar la oportunidad que esos, entonces "nuevos", canales ofrecían. Durante ese periodo la única manera de conseguir los materiales presentados y acceder a los contenidos, era asistiendo y/o consiguiendo las actas que se difundía en soporte impreso o en un $\mathrm{CD}$, distribuidas sólo entre los asistentes. Como la distribución era tan restringida, a esa tipología documental se le denominaba literatura gris o no convencional ${ }^{(4)}$.

Como en otras infinitas facetas de la vida cotidiana y profesional hubo un momento de recesión económica, en el que todavía nos encontramos, y eso afectó doblemente a los congresos. Por un lado, éstos comenzaron a disponer de menos recursos para su organización. De otro, los asistentes potenciales se tuvieron que adaptar a asistir a los mismos con menos dotaciones económicas lo que se trasladó en muchos casos a un intento de contar con maneras alternativas para participar, solventando así el coste de una asistencia durante unos días en un punto geográfico determinado. Los profesionales e investigadores que participan de alguna manera en estos encuentros periódicos ven cómo el número de asistentes físicos ha descendido considerablemente en los últimos años.

Ante ese escenario fueron desarrolladas dos alternativas tecnológicas que promueven la apertura de los encuentros científicos y que hacen que algo que podía haber sido el principio de la desaparición de las reuniones científicas, se convirtiera en opciones para mantener el fin por el que se crearon. Una de las alternativas fue contar con la posibilidad de realizar emisiones abiertas en streaming, en ocasiones con inscripción al evento de manera virtual ${ }^{(5)}$, pero evitando con ellas los costes de desplazamiento; en otras ni tan siquiera con un coste para el visualizador de esos contenidos emitidos, 
a tiempo real o posteriormente. Otra, es la posibilidad que brindan las comúnmente conocidas como redes sociales para un seguimiento de los principales acontecimientos difundidos en ellas ${ }^{(6)}$.

Tras la aparición de esas redes sociales (Twitter, Facebook, Google+, Youtube, entre otras) éstas se incorporan rápidamente y con un efecto de propagación muy rápido, formando en este momento una parte importante de la vida cotidiana ${ }^{(7)}$. Actualmente es difícil pensar en que un congreso que cuente con buena organización y unos contenidos de calidad, no haga un uso de ellas, al menos de alguna red social.

Entre todas las posibles redes sociales, el presente estudio se va a centrar en Tiwtter, que puede ser utilizada en diferentes momentos de un encuentro $\mathrm{y}$, dependiendo del momento en el que se emplee, condicionará el número de los seguidores potenciales y el fin para el que es más oportuno utilizarla en cada etapa: antes, durante la conferencia y después de haber concluido ${ }^{(8)}$. Antes de la conferencia el uso se orienta hacia la difusión del programa, las intervenciones principales, los aspectos que afectan al registro de los asistentes interesados o a la mera información general, como puede ser la localización de la sede o los alojamientos recomendados. La organización del encuentro durante esa fase trata de motivar lo más activamente a la comunidad para la que éste se orienta. Durante la conferencia se pueden anunciar cambios, difundir fotos o resaltar contenidos. Las cuestiones directamente vinculadas con los contenidos más relevantes suelen formar parte de esta fase. Una vez que ha concluido la conferencia se suele agradecer la participación de los asistentes, compartir unas reflexiones finales o aportar estadísticas interesantes. Si procede, es una buena oportunidad para anunciar la celebración del próximo evento.

Generalmente la organización del congreso suele contar con una cuenta en la red social Twitter y a su vez, todos los interesados pueden hacer mención al evento utilizando uno o varios hashtags ${ }^{(9)}$; siguiendo ese proceso el emisor realiza la tarea de categorizar cada uno de los mensajes instantáneos enviados.

En este trabajo se analizará el uso de dos hashtag creados para ser utilizados durante la celebración de dos encuentros convocados en años consecutivos, 2013 y 2014 . Se analiza la información que aportan ambos y con ello se pretende responder a las siguientes cuestiones sobre los eventos analizados, además de manera paralela se pretende ofrecer a las organizaciones de diferentes eventos, una hoja de ruta para el análisis obligado tras concluir un evento en el que se haya usado la red social Twitter. Se pretende dar respuesta a los siguientes interrogantes:

- ¿Existe interacción ente los usuarios, que de manera similar a la mención formal que se recoge en las citas bibliográficas, dejen constancia de las menciones que registran los retuits?

- ¿Hay momentos de una mayor actividad en el uso de los hashtags?

- ¿Tienen los usuarios de Twitter roles diferentes con un potencial interesante como buenos informantes $u$ otros que están bien informados?

- ¿Revelan los términos que los usuarios utilizan un contenido semántico próximo a la temática del congreso?

El interés internacional en el ámbito de ciencias de la salud por analizar la comunicación en este medio social está en auge ${ }^{(10)}$. Se han publicado trabajos sobre el uso de Twitter en reuniones científicas para especialistas en Anestesia (11); Oncología clínica ${ }^{(12)}$; Medicina de urgencias ${ }^{(13,14)}$; Nefrología ${ }^{(15) ; ~ O f t a l m o l o g i ́ a ~(16) ; ~}$ 
Cirugía ${ }^{(17,18)}$; Radiología ${ }^{(19)}$; Promoción de la salud ${ }^{(20)}$; Farmacología ${ }^{(21)}$ o Urología ${ }^{(22,23)}$, por mencionar algunos ámbitos sanitarios.

Si bien, éstas no son las únicas especialidades en las que se está intentado revelar las características de las pautas de comunicación propia de las redes sociales. También se han desarrollado diversas investigaciones para congresos internacionales de especialistas en Educación multimedia (24); Blogeros en la web ${ }^{(1)}$; Lenguaje moderno ${ }^{(25)}$; Web semántica ${ }^{(26)}$ o Community Managers ${ }^{(27)}$.

Frente a esa aproximación internacional, en una esfera de ámbito nacional, ese tipo de labor apenas se ha realizado en congresos nacionales ${ }^{(28)}$. Parece poco oportuno desaprovechar la retroalimentación que se puede lograr tras las reuniones científicas en general, pero con especial énfasis en las vinculadas al ámbito sanitario.

\section{El Encuentro Internacional de Investigación en Cuidados}

Uno de los múltiples encuentros internacionales que se celebran en España está organizado por la Unidad de Investigación en Cuidados de Salud, o Investén-isciii, que pertenece al Instituto de Salud Carlos III y que desde 1996 modela el Encuentro Internacional de Investigación en Cuidados, con el fin de impulsar la cualificación de los profesionales en cuidados, fortalecer la base científica de enfermería y en la provisión de cuidados. En España el Instituto de Salud Carlos III es un importante Organismo Público de Investigación, u OPI, que financia, gestiona y ejecuta la investigación en salud estatal en este país. Investén, como la unidad de investigación que es y que mantiene su adscripción con el mencionado instituto, tiene como objetivo procurar el desarrollo de una estrategia a nivel estatal para fomentar y coordinar la investigación transversal y multidisciplinar en cuidados.

Desde el año mencionado, 1996, y con una periodicidad anual, cada mes de noviembre, los profesionales e investigadores de diferentes disciplinas de la salud presentan sus trabajos a la comunidad científica siendo el momento adecuado para la reflexión, opinión y el intercambio de conocimiento. El contenido de las sesiones tratan, entre otras temáticas, sobre la aplicación de las nuevas tecnologías en la salud; el cáncer; los cuidados de salud en las mujeres, niños y adolescentes; la enfermería y salud mental; los cuidados paliativos; la docencia en enfermería; el envejecimiento y la salud; el género, la salud y las poblaciones vulnerables; la práctica basada en la evidencia; la utilización de la investigación o la medida de los resultados en salud, entre otros.

Además de la presentación de las comunicaciones de los participantes en el encuentro se organizan talleres formativos. En él también se celebran dos sesiones plenarias que son la oportunidad propicia para contar con la presencia de expertos en el ámbito de la salud.

\section{MATERIAL Y MÉTODO}

Se monitorizó la actividad en Twitter durante la celebración del XVII Encuentro Internacional de Investigación en Cuidados celebrado en Lleida, España, durante los días 12 al 15 de noviembre de 2013 y el XVIII Encuentro Internacional de Investigación en Cuidados celebrado en Vitoria-Gasteiz, también en España, entre los días 11 y 14 de noviembre de 2014. Para ello se recopilaron todos los tuits que contuvieran los hashtags oficiales del encuentro de cada año (\#inves13 y \#inves14), 
desde el día inicial martes a partir de las 15:00 horas hasta el último día, el viernes, difundidos como máximo a las 15:59 horas.

La recogida de datos se realizó empleando la tecnología de Opileak ${ }^{(29)}$ desarrollada en Java, obteniendo como resultado todos los tuits que incluyeran los hashtags, considerando los retuits y una breve información sobre los usuarios que los utilizaron. Tras ese proceso de recolección, se procedió a anonimizar todos los datos para su análisis. Con el fin de facilitar la interpretación y haciendo uso de la metodología propia del Análisis de Redes Sociales, o ARS, se generaron sendos grafos en los que aparecen, entre otros, los nodos participantes y las relaciones que ocurrieron entre ellos. Con las interacciones entre usuarios se refleja la estructura social que puede ser analizada para así revelar su dinámica. Paralelamente, se realizó un estudio descriptivo de todos los registros producidos en el período de estudio y por último, se desarrolló un análisis semántico del contenido de los tuits recogidos. Muchos trabajos de los incluidos en el apartado dedicado a la recolección de trabajos previos realizan una clasificación de los mensajes, si bien es un método con un alto grado de intervención humana y puede ser igual o más revelador conocer el contenido semántico de los mensajes.

Para todo ello se utilizaron diferentes programas. Así para el análisis descriptivo de los datos se trabajó con la hoja de cálculo de Microsoft Excel 2007, para el análisis de redes se utilizó Pajek y para el análisis semántico se empleó el programa estadístico R.

Los usuarios de Twitter además de poder asignar un hashtag relacionado con el contenido o que lo defina, pueden mencionar a otros usuarios. Eso hace que se disponga de información de la red que se crea por las menciones, que dejan constancia de relaciones asimétricas. Eso quiere decir que un usuario $A$ de Twitter puede mencionar a un usuario $B$, si bien es probable que ese segundo usuario no mencione en alguno de sus tuits al primero (siendo poco probable que sea una relación recíproca).

Los hashtags formalmente quedan recogidos en un tuit tras el uso del símbolo \#, mientras que las menciones a otros usuarios se registran empleando el signo @. Así, un hipotético tuit con dos menciones podría ser algo similar a "@usuarioA RT: @usuarioB: presentando con @usuarioC una comunicación en \#inves14”. El tipo de mensaje sería un retuit y la relación basada en menciones quedaría registrada indicando que el usuario1 ha mencionado al usuario2, y también el usuario1, ha mencionado al usuario3. Con todas las menciones que los usuarios realizaron durante los cuatro días, se obtuvo la matriz con la que se trabajó que tenía forma bidimensional, era una matriz de modo 1 integrada por relaciones que según su dirección hacen que la matriz sea a su vez asimétrica y ponderada.

Para el análisis semántico se trabajó con todos los tuits difundidos durante los días y las horas definidas en este apartado metodológico. Para obtener la nube de palabras que aporta de una forma sencilla ${ }^{\left({ }^{30}\right)}$ el contenido semántico de los encuentros, en primer lugar se borró toda la información que identifica hashtags (\#), retuits (RT), usuarios (@), enlaces y cualquier otro signo de puntuación. Tras ello, se procedieron a eliminar las palabras vacías, entre las que están los artículos o preposiciones, por mencionar algunas. Todas las letras se convirtieron tipográficamente en minúsculas. Concluidos esos procesos, en la nube los términos más frecuentes aparecen en una posición central, con un mayor volumen y un tono de color diferente. 


\section{RESULTADOS}

En el encuentro celebrado durante el año 2013 se contó con un total de 498 participantes. Después de la llamada para recibir propuestas, la organización del congreso recibió 585 resúmenes, de los que fueron aceptados 473. Los mismos datos para el encuentro del año 2014 indican que fue superior el número de participantes, un total de 527, si bien los resúmenes recibidos fueron menos numerosos, tan solo 510. De ellos finalmente se aceptaron 433.

Para confirmar si existe interacción ente los usuarios, tras la constancia que dejan en los mensajes, se comienza aportando el dato de la actividad registrada en Twitter en los dos años que ascendió a un total de 3734. De ellos fueron originales 1640 y 2094 utilizaron los que otros usuarios ya habían difundido en la red, es decir, fueron retuiteados de manera similar al proceso de citas bibliográficas en documentos científicos. La distribución por años tras el uso del hashtag \#inves13 e \#inves14 (en el Gráfico 1 series en naranja y verde, respectivamente), en 2013 los contenidos originales fueron superiores a los retuiteados (973 frente a 750). Revisando el uso el hashtag del encuentro del año 2014, el proceso se invirtió y el proceso de retuitear fue mucho más frecuente (1344) que el de difundir mensajes originales (667).

Gráfico 1. Mensajes originales y retuiteados que utilizaron los hashatags del encuentro

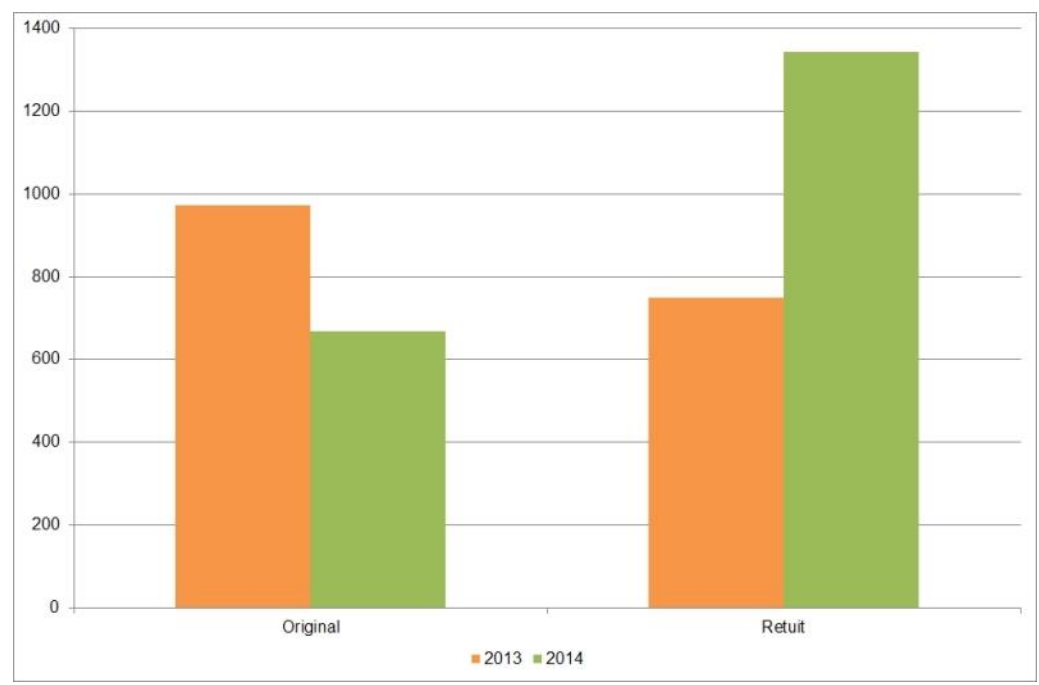

A lo largo de los cuatro días, el uso de hashtags pasa por momentos de diferente volumen de actividad. Este hecho es relevante para una oportuna difusión de cuestiones consideradas como claves para cualquier organización de un congreso, es de gran interés conocer los días y los momentos del día en los que hay una mayor actividad en el uso de los haghtags. El encuentro anual que se está analizando se tiende a celebrar durante unas fechas muy similares y su programa se organiza siempre durante cuatro días, desde el martes hasta el viernes de la misma semana. Durante los dos encuentros estudiados parece que el primer día, el martes, tiene un tráfico de mensajes en la red poco numeroso. Se puede afirmar que los otros tres son los días clave para este encuentro.

Los momentos con más actividad durante el año 2013 se sitúan a las 17 horas del martes, a las 12 horas el miércoles, a las 9 horas el jueves y el viernes de nuevo, a las 12 horas. El segundo año, el 2014, la hora más activa del martes fue las 17 horas, el miércoles a las 11 de la mañana, el jueves a las 15 horas y el viernes tanto a las 10 
horas como a las 15 horas. Para tener una perspectiva detallada de los mensajes que utilizaron los hashtags por horas y días de la semana se debe consultar el Gráfico 2

Gráfico 2. Mensajes que utilizaron los hashtags del encuentro por día de la semana y hora del día

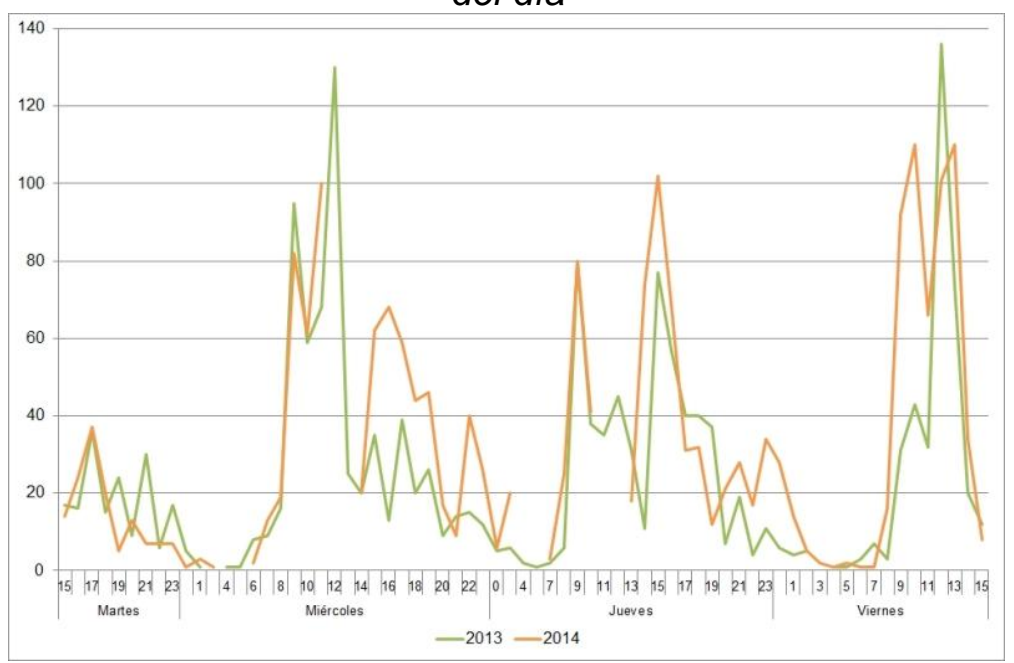

Centrando la atención en las diferencias entre mensajes originales y retuiteados por día de la semana, en el año 2013 el día que más mensajes originales fueron enviados fue el miércoles, con un dato muy próximo a los enviados el jueves. En el año 2014, para esos mismos mensajes originales, con valores ligeramente inferiores a los del año previo, el viernes fue el día con más mensajes enviados, seguido del miércoles. En cualquiera de los dos años, el martes de nuevo queda a una distancia considerable del resto de días.

La ya comentada dinámica de envío de mensajes retuiteados en 2014, se vuelve a confirmar revisando el Gráfico 3. En ese año y con valores muy próximos, la actividad del miércoles y jueves, alcanzó un importante nivel de mensajes, con un valor medio próximo a los 450 mensajes por día. Aunque se mantiene la comentada distancia con el martes, sí que para los mensajes retuiteados en ese año, los así difundidos alcanzan el valor más alto para todos los tipos de mensajes emitidos. En el año 2013, el énfasis se puso en los contenidos originales y en cualquier día de la semana las diferencias son similares a los mensajes retuiteados, si bien, los valores absolutos de mensajes retuiteados son moderadamente menores a los originales. 
Gráfico 3. Mensajes originales y retuiteados que utilizaron los hashtags del encuentro por día de la semana

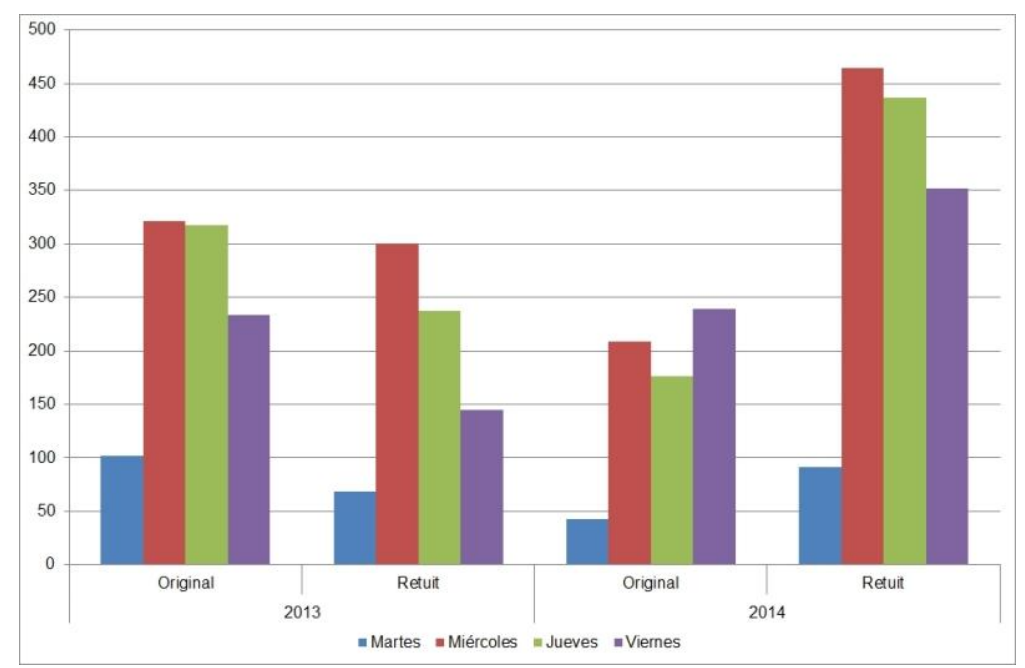

Aunque hay una cierta actividad durante todo el día, tomando en cuenta las horas de descanso de los usuarios de Twitter, este estudio se centra en el rango del día comprendido desde las 8 a las 20 horas (Gráfico 4). Eso supone un tráfico de 3260 mensajes frente a los 3734 totales. Los mensajes originales del año 2013 se difunden en gran medida a las 12 horas y a las 9 . Esa misma hora fue la más frecuente en el año 2014. En el caso de los mensajes retuiteados de nuevo es a las 12 horas el momento del día con más mensajes enviados de esa forma en el año 2013, no habiendo una hora muy frecuente para los retuits en el año 2014. La frecuencia se sitúa en valores muy similares desde las 9 a las 11 y desde las 13 a las 18 horas.

Gráfico 4. Mensajes originales y retuiteados que utilizaron los hashtags del encuentro por hora del día (de 8 a 20 h.)

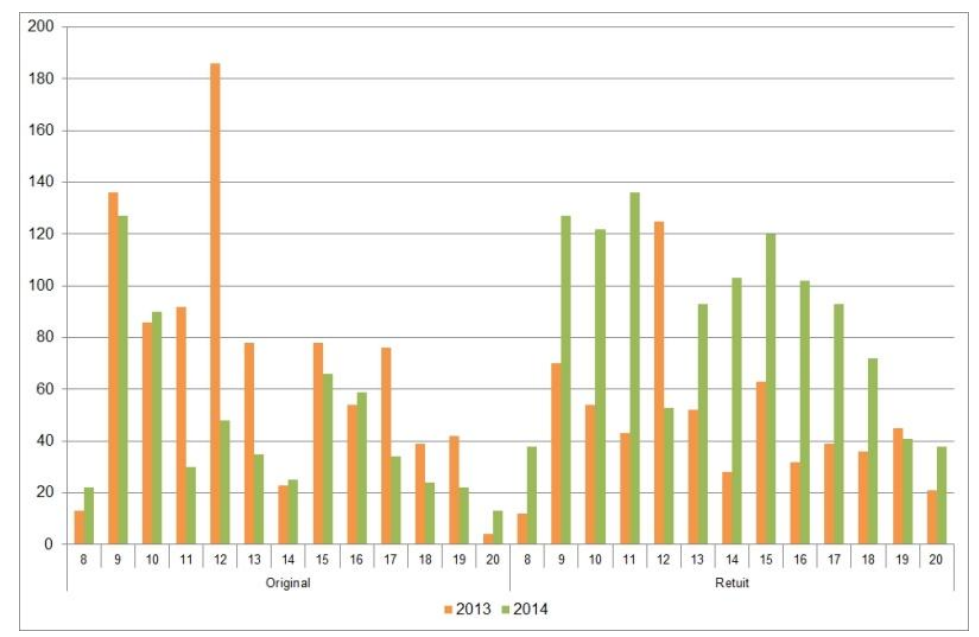

La actividad en la red Twitter es la de sus usuarios. Asimismo cada usuario además de un volumen de actividad diferente, durante los días del congreso va a tomar un rol cambiante. Aunque su rol puede ser medido desde diferentes perspectivas, es de interés identificar qué usuarios son buenos informadores frente a los que están bien informados. 
Durante los cuatro días que duró el congreso en el año 2013, 332 usuarios diferentes utilizaron el hashtag \#inves13 y en 2014 casi se duplicó el número, 612 usuarios. Con esas cantidades, es probable que no todos asumieran el mismo rol en la red de menciones y finalmente algunos sean buenos informadores, mientras que otros usuarios han estado bien informados durante el congreso.

Para identificar esos posibles roles, se hace uso del algoritmo conocido como HITS (acrónimo de Hyperlink-Induced Topic Search) que es frecuentemente utilizado para detectar estructuras en el ciberespacio ${ }^{(1)}$. Con él se identifican dos tipos de nodos: hubs y autoridades o authorities ${ }^{(31,32)}$. Jon Kleinber definió que un nodo, aunque en el trabajo original se hace mención específica a una página web, es un buen hub, si apunta a buenas autoridades (según las características de sus enlaces salientes). Y en ese sentido un nodo es una buena autoridad, si es apuntado por buenos hubs, considerando para ello las características de sus enlaces entrantes. Este mecanismo de refuerzo mutuo es descrito por la pareja de relaciones calculadas como sigue ${ }^{(33)}$ :

$$
\begin{aligned}
\lambda y_{i} & =\sum_{j: j \rightarrow i} x_{j}=\sum_{j} A_{j i} x_{j}=\left(A^{t} x\right)_{i} \\
\mu x_{i} & =\sum_{j: i \rightarrow i} y_{j}=\sum_{j} A_{i j} y_{j}=(A y)_{i}
\end{aligned}
$$

Ambas pueden ser reescritas desde la forma de ecuaciones simples de valores propios para las sustituciones $x$ e $y$ :

$$
\begin{aligned}
& \lambda \mu x_{i}=\left(A A^{t} x\right)_{i} \\
& \lambda \mu y_{i}=\left(A^{t} A y\right)_{i}
\end{aligned}
$$

Las puntuaciones de hub y autoridad son vectores propios de las matrices $A A^{t}$ y $A^{t} A$. Siendo ambas simétricas, mientras que A puede ser simétrica o no. Las puntuaciones de $x$ e $y$ corresponden con los vectores propios de esas matrices.

La red de los 332 usuarios que utilizaron el hashtag \#inves13, está formada por un gran componente fuerte que incluye el $97 \%$ de los nodos. Como se puede ver en el área derecha del grafo incluido en la Figura 1, hay nodos que no mencionaron a ningún otro usuario y por eso quedan aislados de ese gran componente. Algunos usuarios en ocasiones se automencionan en los tuits, algo similar a lo que ocurre con las autocitas, y ese efecto en la red queda representado con una flecha cuyo inicio y destino es el mismo nodo. En color lila quedan identificados los usuarios de Twitter que son buenas autoridades, por lo tanto, buenos informantes. En color verde aparecen aquellos que son buenos hubs, y por ello, están bien informados. Pero además hay un tercer rol, aquellos usuarios que además de estar bien informados, son buenos informantes, representados en color rojo. 
Figura 1. Red de menciones entre los usuarios que utilizaron el hashtag \#inves 13

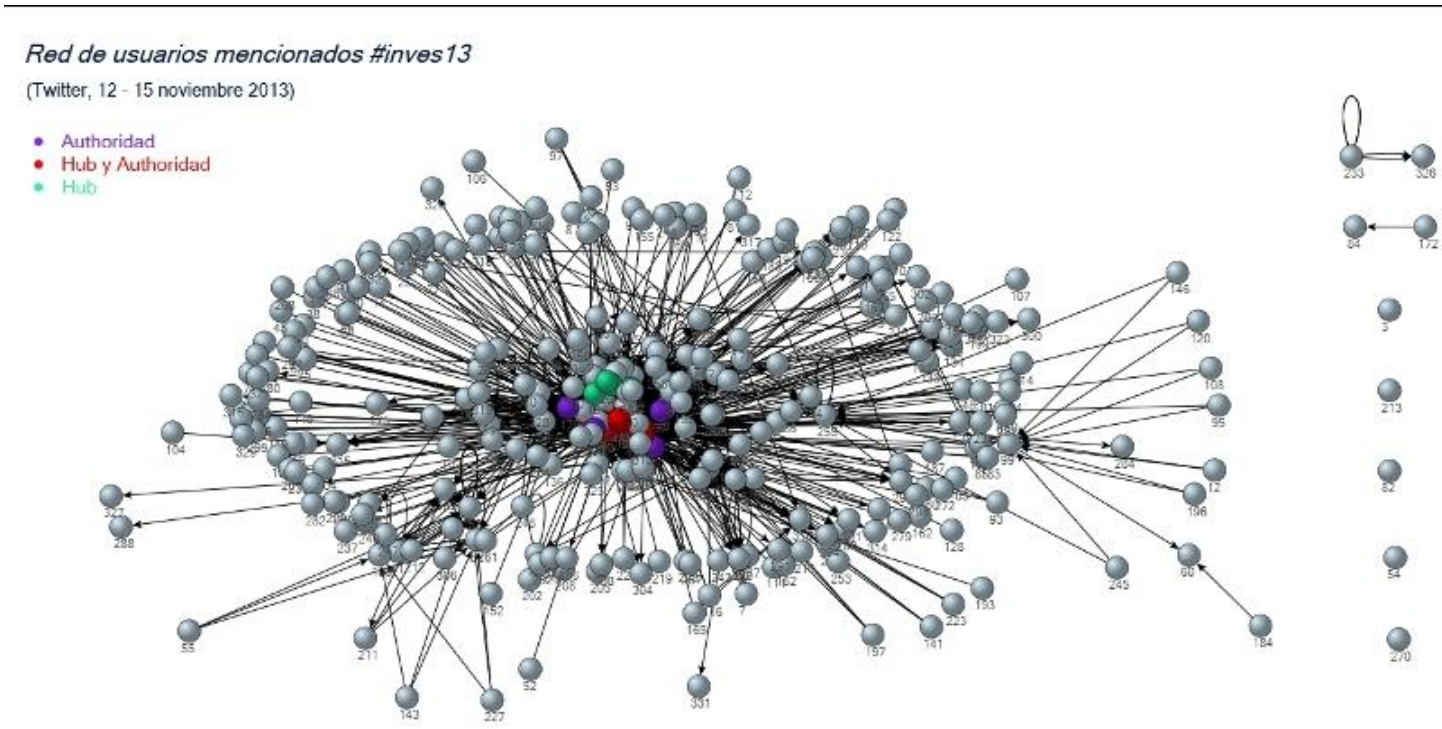

La red de los 612 usuarios que utilizaron el hashtag \#inves14, tiene una topografía diferente y no mantiene una estructura centro-periferia como quedaba la red de las menciones entre los usuarios de \#inves13. En el grafo recogido en la Figura 2, aunque el centro se mantiene, en la periferia hay dos usuarios claves (489 y 536) que conectan ésta con ese centro. De nuevo en esta red tanto los usuarios hubs como autoridades están en la parte central de la red. Con un valor muy similar, el porcentaje de usuarios en el componente fuerte es de un $98 \%$.

Figura 2. Red de menciones entre los usuarios que utilizaron el hashtag \#inves13

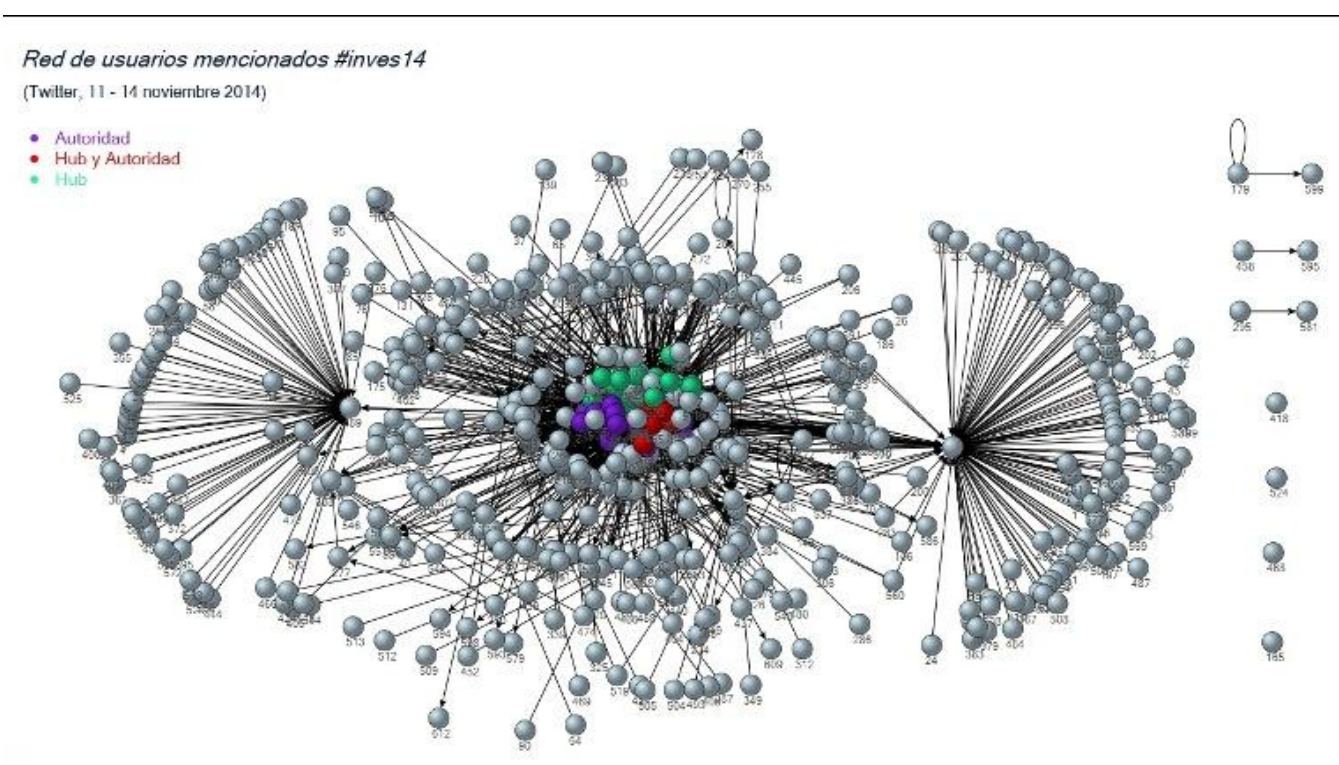

En la Tabla I se recoge el grupo de medidas propias del ARS que sintetizan las principales propiedades de ambos grafos ${ }^{(34)}$. 
Tabla I. Principales indicadores del análisis de las redes de menciones entre usuarios

\begin{tabular}{|c|c|c|}
\hline Propiedades & Hinves13 & Hinves14 \\
\hline Nodos & 332 & 612 \\
\hline Nodos sólo mencionados & 60 & 35 \\
\hline Nodos presentes en ambas redes & 62 & \\
\hline Flechas (relaciones dirigidas) & 1898 & 2515 \\
\hline Número de automenciones (loops) & 105 & 82 \\
\hline Densidad (con loops) & 0.0172 & 0.00671 \\
\hline Grado medio & 11.4337 & 8.2189 \\
\hline Componentes débiles & 8 & \\
\hline Tamaño del componente débil más grande & $323(97.289 \%)$ & $602(98.366 \%)$ \\
\hline Componentes fuertes & 262 & 533 \\
\hline Tamaño del componente fuerte más grande & $65(19.578 \%)$ & $78(12.745 \%)$ \\
\hline Diámetro & 9 & \\
\hline Camino más largo & 55 a 60 & 4 a 483 \\
\hline
\end{tabular}

Además de los usuarios, el valor principal de las redes sociales es conocer el contenido principal para el que se están utilizando. Como ya se avanzó en la introducción, algunos trabajos previos clasificaban el contenido de los mensajes pero ese proceso se realiza por personas y por lo tanto es bastante subjetivo. Para conseguir más objetividad, el análisis de términos es una aproximación al contenido semántico que define el interés del encuentro en Twitter; y la nube de términos una representación gráfica oportuna para visualizar éstos.

Los diez términos más frecuentes tras el uso del hashtag \#inves13 (Figura 3) son Lleida, investigación, cuidados, salud, congreso, mesa, encuentro, premio, enfermeras y tuit. Para \#inves14 (Figura 4), algunos se repiten y otros se incorporan como, enfermería, mejor, cuidarte, edcuidando, investigan, enfermeriavisible, pacientes.

Figura 3. Nube de términos incluidos en los mensajes que utilizaron el hashtag \#inves13

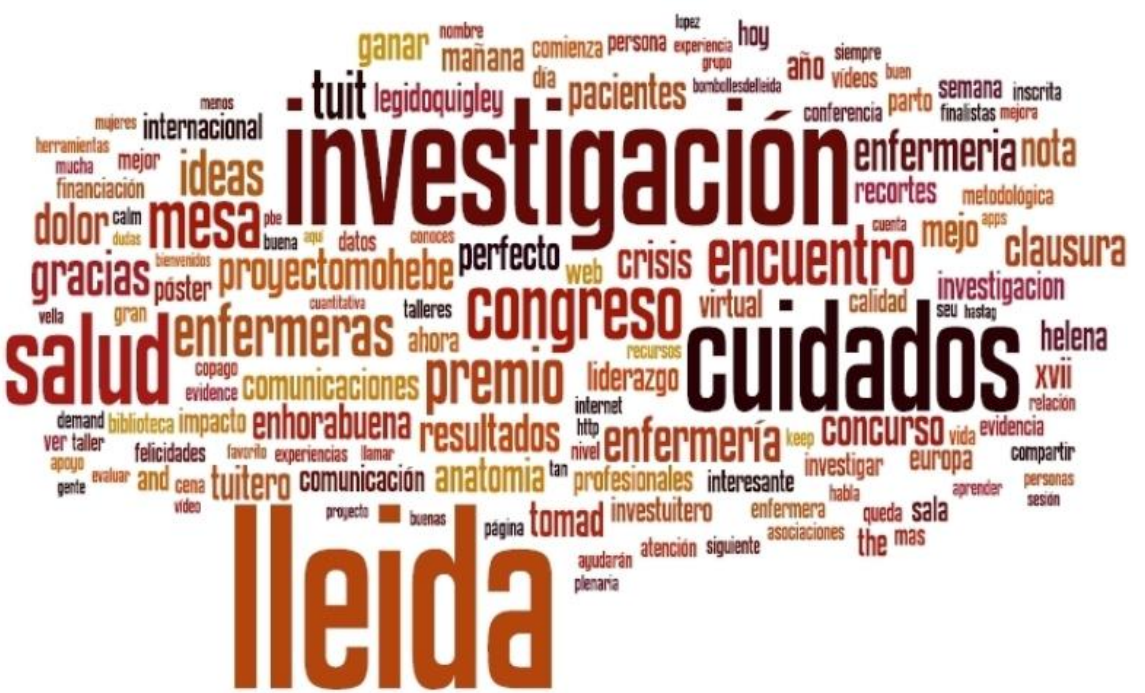


Figura 4. Nube de términos incluidos en los mensajes que utilizaron el hashtag \#inves14

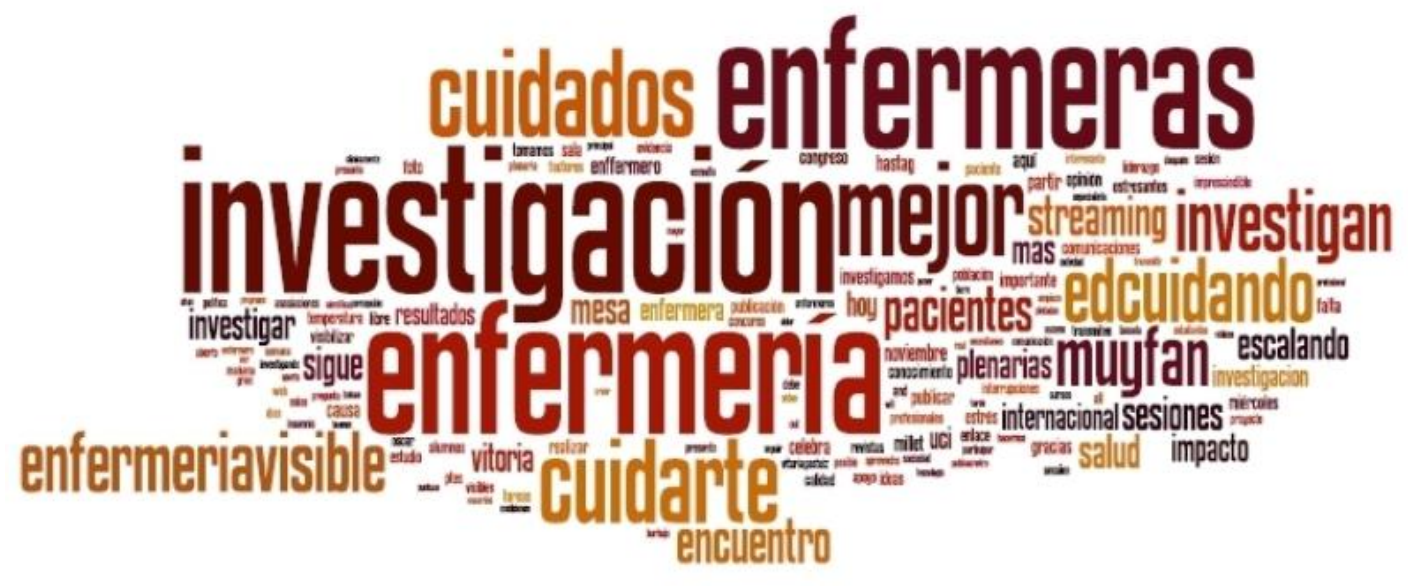

De igual manera que los usuarios se relacionan con otros mediante las menciones, los términos aparecen en un contexto que les sitúa simultáneamente con otros términos. La proximidad con otros términos se identifica con la correlación entre ambos y es una medida que aporta la suficiente información sobre el contexto en el que se utilizan.

Para los cuatro términos más frecuentes en la figura 3, las palabras con mayor correlación para Lleida son acoger, evento y orgullo. Para investigación son cuidados, internacional y conoces. Para cuidados son investigación, internacional y conoces. Para salud son impacto, helena, crisis, legidoquigley. En la tabla II se pueden consultar los valores de correlación para cada término.

Tabla II. Asociación de palabras con los términos más frecuentes en los mensajes que utilizaron el hashtag \#inves 13

\begin{tabular}{l|l|l|l|l|l|l|l|}
\hline Ileida & Corr. & investigación & Corr. & cuidados & Corr. & salud & Corr. \\
\hline acoger & 0.39 & cuidados & 0.53 & investigación & 0.53 & impacto & 0.44 \\
\hline evento & 0.39 & internacional & 0.37 & internacional & 0.38 & helena & 0.41 \\
\hline orgullo & 0.35 & conoces & 0.34 & conoces & 0.36 & $\begin{array}{l}\text { crisis } \\
\text { legidoquigle }\end{array}$ & 0.40 \\
& & & & & & legido \\
\hline
\end{tabular}

En el caso de los cuatro términos más frecuentes en la figura 4, las palabras con mayor correlación para investigación son cuidados, escalando, internacional. Para enfermeras son cuidarte, investigan, mejor. Para enfermería son crear, tecnología, transmitir. Para mejor son cuidarte, muyfan, investigan. La tabla III incluye los valores de correlación.

Tabla III. Asociación de palabras con los términos más frecuentes en los mensajes que utilizaron el hashtag \#inves 14

\begin{tabular}{l|l|l|l|l|l|l|l|}
\hline investigación & Corr. & enfermeras & Corr. & enfermeria & Corr. & mejor & Corr. \\
\hline cuidados & 0.53 & cuidarte & 0.69 & crear & 0.31 & cuidarte & 0.94 \\
\hline escalando & 0.44 & investigan & 0.66 & tecnología & 0.31 & muyfan & 0.78 \\
\hline internacional & 0.39 & mejor & 0.65 & transmitir & 0.31 & investigan & 0.76 \\
\hline
\end{tabular}




\section{DISCUSIÓN}

El análisis del uso de Twitter durante los XVII y XVIII Encuentros Internacionales de Investigación en Cuidados es una labor clave para conocer las características propias de este medio de comunicación. Los datos monitorizados desde Twitter se recopilaron siguiendo un método cualitativo de recogida de datos que permite conocer el uso de las redes sociales pudiendo alternarse otros como la encuesta, entrevista $u$ observación de los participantes en los eventos ${ }^{(28)}$. La mejor opción sería combinar más de un método para recopilar los datos de usuarios registrados y paralelamente disponer de sus cuentas individuales de Twitter, dato que se puede solicitar en el momento de la inscripción ${ }^{(17)}$.

Durante el encuentro celebrado en el año 2014 hubo más participantes inscritos pero se recibieron menos propuestas de trabajo y eso tuvo como consecuencia que menos trabajos también fueran finalmente aceptados con respecto a la cantidad de los que lo fueron en 2013. En ambos años, el número de mensajes aumentó, de 1723 a 2011, y es una tendencia habitual que el uso de la red social Twitter crezca con el paso de los años ${ }^{(12)}$.

En ambos encuentros se promocionó el uso de la red social Twitter y se premió a la persona más activa en términos de tuits que a su vez estuviera inscrita en los encuentros. También se premió a la persona que difundiera un único tuit y que éste fuera el más retuiteado. Tanto en el año 2013 como en el 2014 esa promoción se orientó en los mismos términos pero fue durante el segundo año cuando parece que influyó en que los retuits superaran a los mensajes originales. Así el año 2013 se caracteriza por una mayor proporción de originales y 2014 por la alta difusión de mensajes retuiteados. En los años más actuales la dinámica de retuitear es más habitual que en los años previos ${ }^{(25)}$ y esa práctica se hace por personas sobre las que se puede afirmar que pertenecen a una misma comunidad ${ }^{(26)}$.

Los momentos de mayor actividad en el uso de hashtags coinciden en ocasiones para \#inves13 e \#inves14 y hay otras situaciones de alta actividad que son únicas en cada encuentro. Se puede afirmar que en líneas generales los mensajes se envían de manera arbitraria ${ }^{(24)}$, aunque en ambos años ocurren momentos con un cierto paralelismo en relación con la cantidad de mensajes enviados.

La organización del congreso, según lo que se refleja Twitter, debe tomar en cuenta que los contenidos del primer día parecen tener un impacto en la red social menor si se comparan con el resto de jornadas. Según el criterio basado en el volumen de mensajes, los principales días son los miércoles y jueves; a su vez, el miércoles registra más actividad para cualquier tipo de mensaje en ambos años. Por todo ello parece que es habitual que en los días centrales del congreso se concentre la mayor actividad en esta red social ${ }^{(35)}$.

Durante las diferentes horas del día en el periodo bianual se retuitea habitualmente, teniendo los originales momentos que alcanzan un cierto volumen también a considerar. Frente a esos picos de envíos de mensajes originales, los retuits fueron enviados con una frecuencia muy similar durante los cuatro días en los que se celebra el congreso.

Desde el primer año al segundo, el número los usuarios que utilizó el hashtag casi se llegaron a duplicar. Se podría afirmar, considerando que la serie tiene un rango de 
dos años, que parece existir una tendencia creciente a realizar un seguimiento 2.0 en este tipo de encuentros según se van organizando congresos en años posteriores ${ }^{(25)}$. Si se quisiera lograr una amplia difusión de aspectos claves del evento, como sería el caso del anuncio del siguiente encuentro por ejemplo, la organización debería mantener contacto con aquellos usuarios que adoptan un rol como autoridad. La propia organización, en aras de detectar posibles intereses, debiera además realizar la acción de seguir en Twitter a aquellos usuarios que son buenos hubs. Es lógico también considerar que a cualquier usuario normal con interés en la materia del congreso, debiera establecer una relación como seguidor de aquellos usuarios que en ediciones pasadas fueron simultáneamente hubs y autoridades.

Desde el punto de vista del Análisis de Redes Sociales, las redes como Twitter se pueden clasificar como "redes sin escala" o mostrar como "el fenómeno de mundo pequeño", propiedades habituales en la esfera web y los blogs ${ }^{(1)}$.

La propuesta del premio podría haber influido en el cambio de la estructura de la red en el segundo año. En el 2014 los que conectan el centro de la red de menciones con la periferia se corresponden con los dos candidatos al reconocimiento porque un único tuit de ellos fue el que más retuits alcanzó. La comunicación en sí es un acto muy dinámico y las redes creadas tras diferentes eventos evolucionan, aunque tiendan a mantener estructuras similares. Eso no ocurre exclusivamente con las redes de los congresos y también lo hace en las redes socio-políticas ${ }^{(36)}$.

Los términos que los usuarios utilizan en sus mensajes, sin considerar las menciones o los hashtags utilizados, y las palabras asociadas a ellos tienen la capacidad de definir el eje principal del congreso. Así en Twitter queda la constancia en firme de las ciudades en las que se celebró el congreso (Lleida o Vitoria), la temática del encuentro (investigación en cuidados) y su carácter (internacional).

Principalmente, aunque no exclusivamente, Twitter es una herramienta poderosa para extender el contenido de las reuniones científicas, más allá del difundido durante un tiempo y lugar determinados (23). Se añade la característica de esencial, especialmente si se piensa en la información necesaria para establecer buenas estrategias de difusión en la organización de eventos futuros.

\section{CONCLUSIÓN}

El análisis del uso de Twitter durante los encuentros científicos es una labor que puede aportar información de gran valor. Con ese proceso se pueden revelar pautas en la comunicación como la interacción entre los usuarios, los momentos en los que hay una mayor actividad, los diferentes roles que aportan los usuarios en la red social y el análisis semántico de los términos que utilizaron. Los organizadores de congresos científicos españolas debieran poner en marcha estrategias para aprovechar esta oportunidad y con la hoja de ruta incluida en este trabajo, cuentan con las pautas esenciales para trabajar hacia ese objetivo.

\section{REFERENCIAS}

1. Java $A$, Song $X$, Finin T, Tseng B. Why we twitter: understanding microblogging usage and communities. Proceedings of the 9th WebKDD and 1st SNA-KDD 2007 workshop on Web mining and social network analysis (WebKDD/SNA-KDD '07).New York: ACM; 2007. Disponible en: 
http://ebiquity.umbc.edu/_file_directory_papers/369.pdf. [Consultado el 29 de mayo de 2015]

2. Mena Muñoz S. Medición del tiempo de efectividad de los tuits. Interés y vida de la información en la era de las redes sociales. In: Vicente Mariño V, González

Hortigüela T, Pacheco Rueda M, editors. Investigar la Comunicación hoy. Revisión de políticas científicas y aportaciones metodológicas: Simposio Internacional sobre Política Científica en Comunicación. 2013. p. 173-84. Disponible en:

http://dialnet.unirioja.es/descarga/articulo/4228715.pdf. [Consultado el 1 de junio de 2015]

3. Marqués Sánchez P, González Pérez ME, Agra Varela Y, Vega Núñez J, Pinto Carral A, Quiroga Sánchez E. El análisis de las redes sociales. Un método para la mejora de la seguridad en las organizaciones sanitarias. Revista Española de Salud Pública 2013;87 (3): 209-19.

4. Moreno-Torres Sánchez R. El acceso a la literatura gris: actas de congresos y tesis doctorales. Boletín de Anabad 1986; 36 (4): 671-96.

5. Escudero Sánchez M, Fernández Cáceres JL, Malgosa Sanahuja JM.

Retransmisiones y congresos en Internet. Contenidos y Aspectos Legales en la Sociedad de la Información (CALSI), Valencia (Spain), 22-23 October. 2002.

Disponible en: http://eprints.rclis.org/4314/ [Consultado el 5 de junio de 2015]

6. Goff DA, Van Den Bergh D. Twitter: A tool to improve healthcare professionals' awareness of antimicrobial resistance and antimicrobial stewardship. South African Medical Journal 2015; 105 (5): 427-30.

7. Purita G. OBS Social 2015: análisis de las tendencias de uso y participación en las redes sociales a nivel mundial en España. Online Business School; 2015.

Disponible en: http://recursos.anuncios.com/files/681/25.pdf [Consultado el 20 de mayo de 2015]

8. Reinhardt W, Ebner M, Beham G, Costa C. How people are using Twitter during conferences. In: Hornung-Prähauser V, Luckmann M, editors. Proceedings of 5th EduMedia conference: Creativity and innovation competencies on the Web.Salzburg: 2009. p. 145-56.Disponible en: http://www.salzburgresearch.at/wpcontent/uploads/2010/12/Tagungsband_EduMedia_2009_04_01_formatiert_v15.pdf [Consultado el 3 de junio de 2015]

9. Twitter Inc. Centro de Ayuda de Twitter | Bienvenido a Twitter. 2015. Disponible: http://www.twitter.com [Consultado el 1 de junio de 2015]

10. Djuricich AM, Zee-Cheng JE. Live tweeting in medicine: 'Tweeting the meeting'. International Review of Psychiatry 2015; 27 (2): 133-9.

11. McKendrick DR, Cumming GP, Lee AJ. Increased use of Twitter at a medical conference: a report and a review of the educational opportunities. Journal of Medical Internet Research 2012;16 (4).

12. Chaudhry A, Glod, M, Gillman M, Miller RS. Trends in Twitter use by physicians at the American Society of Clinical Oncology annual meeting, 2010 and 2011. Journal of Oncology Practice 2012; 8 (3): 173-8.

13. Roland D, May N, Body R, Carley S, Littley MD. Are you a SCEPTIC? SoCial mEdia Precision \& uTility In Conferences. Emergency Medicine Journal 2014; 32 (5): 412-3.

14. Neill A, Cronin JJ, Brannigan D, O'Sullivan R, Cadogan M. The impact of social media on a major international Emergency Medicine Conference. Emergency Medicine Journal 2014; 31 (5): 401-4.

15. Desai T, Shariff A, Shariff A, Kats M, Fang X, Christiano C, et al. Tweeting the meeting: an in-depth analysis of Twitter activity at Kidney Week 2011. PLOS one 2012; 7 (7): e40253. 
16. Micieli R, Mlcieli JA. Twitter as a tool for ophthalmologists. Canadian Journal of Ophthalmology/Journal Canadien d'Ophtalmologie 2012; 47 (5): 410-3.

17. Cochran A, Kao LS, Gusani NJ, Suliburk JW, Nwomeh BC. Use of Twitter to document the 2013 Academic Surgical Congress. Journal of Surgical Research 2015; 190 (1): 36-40.

18. Logghe H, Maa J, Schwartz J. Twitter usage at Clinical Congress rises markedly over two years. Bulletin of the American College of Surgeons 2013; 98 (2): 22-4.

19. Hawkins CM, Duszak R, Rawson JV. Social media in radiology: early trends in Twitter microblogging at radiology's largest international meeting. Journal of the American College of Radiology 2014; 11 (4): 387-90.

20. Anderson G, Gleeson S, Rissel C, Wen LM, Bedford K. Twitter tweets and twaddle: Twittering at AHPA's National Health Promotion Conference. Health Promotion Journal of Australia 2014; 25 (2): 143-6.

21. Award NI, Cocc C. Use of Twitter at a major national pharmacy conference. American Journal of Health-System Pharmacy 2015; 72 (1): 65-9.

22. Matta R, Doiron C, Leveridge MJ. The dramatic increase in social media in Urology. Journal of Urology 2014; 192 (2): 494-8.

23. Wilkinson SE, Basto MY, Perovic G, Lawrentschuk N, Murphy DG. The social media revolution is changing the conference experience: Analytics and trends from eight international meetings. BJU International 2015; 115 (5): 839-46.

24. Ebner M, Reinhardt W. Social networking in scientific conferences - Twitter as tool for strengthen a scientific community. Proceedings of the 1st International Workshop on Science 2.0 for TEL at the 4th European Conference on Technology Enhanced Learning (EC-TEL'09). 2009.

25. Weller K, Dr"ge E, Puschmann C. Citation analysis in Twitter: approaches for defining and measuring information flows within tweets during scientific conferences. Proceedings of the 1st Workshop on. Making Sense of Microposts (MSM2011). 2011.

26. Letierce J, Passant A, Decker S, Breslin JG. Understanding how Twitter is used to spread scientific messages. Web Science Conference, April 26-27. Raleigh: 2010.

27. Jussila J, Huhtamäki J, Kärkkäinnen H, Still K. Information visualization of Twitter data for co-organizing conferences. Proceedings 17th International Academic MindTrek Conference on International Conference on Making Sense of Converging Media, October 1-4.Tampere: ACM; 2013. p. 139-45.

28. Traver Vall,s $P$, Cobarsí-Morales J. El uso de Twitter en los congresos profesionales de información y documentación: Estudio de caso. Revista General de Información y Documentación 2012; 22: 349-65.

29. Opeliak. 2015. Disponible en: http://www.opileak.es [Consultado el 15 de mayo de 2015]

30. Olmeda Gómez C. Visualización de Información. El Profesional de la Información 2014; 23 (3): 213-9.

31. Kleinberg JM. Authoritative Sources in a Hyperlinked Environment. ACM-SIAM Symposium on discrete algorithms 1998. Disponible en:

http://www.cs.cornell.edu/home/kleinber/auth.pdf [Consultado el 04 de junio de 2015

32. Kleinberg JM. Hubs, Authorities, and Communities. ACM Computing Surveys 1999; 31 (4): 3.

33. Perra N, Fortunato S. Spectral centrality measures in complex networks. Physical Review E 2008; 78 (36107): 36107-1-36107-10.

34. Wasserman S, Faust K. Social network analysis: methods and applications.

Cambridge, New York: Cambridge University Press; 1994.

35. Mishori R, Levy B, Donvan B. Twitter Use at a Family Medicine Conference.

Family Medicine 2014; 46 (8): 608-14. 
36. Del Val E, Rebollo M, Botti V. Does the type of event influence how user interactions evolve on Twitter? PLOS one 2015; 10 (5): e0124

Recibido: 24 de julio 2015; Aceptado: 26 de agosto 2015 